\title{
Expression pattern of REIC/Dkk-3 in various cell types and the implications of the soluble form in prostatic acinar development
}

\author{
KAI ZHANG ${ }^{1}$, MASAMI WATANABE ${ }^{1,2,3}$, YUJI KASHIWAKURA ${ }^{1}$, SHUN-AI LI $^{1,2}$, KOHEI EDAMURA ${ }^{3}$, \\ PENG HUANG $^{1,2}$, KEN YAMAGUCHI ${ }^{2,3}$, YASUTOMO NASU ${ }^{3}$, YASUYUKI KOBAYASHI ${ }^{3}$, \\ MASAKIYO SAKAGUCHI ${ }^{4}, \mathrm{KAZUHIKO} \mathrm{OCHIAI}^{1}$, HIROSHI YAMADA $^{5}, \mathrm{KOHJI}$ TAKEI $^{5}$, \\ HIDEO UEKI ${ }^{3}$, NAM-HO HUH ${ }^{4}$, MING LI $^{6}$, HARUKI KAKU ${ }^{1,2,3}$, \\ YANQUN NA ${ }^{7}$ and HIROMI KUMON $\mathrm{N}^{1,2,3}$
}

\begin{abstract}
${ }^{1}$ Innovation Center Okayama for Nanobio-Targeted Therapy, ${ }^{2}$ Center for Gene and Cell Therapy, Departments of ${ }^{3}$ Urology,
${ }^{4}$ Cell Biology, and ${ }^{5}$ Neuroscience, Graduate School of Medicine, Dentistry and Pharmaceutical Sciences, Okayama University, Okayama, Japan; ${ }^{6}$ Department of Urology, Peking University School of Oncology, Beijing Cancer Hospital and Institute; ${ }^{7}$ Department of Urology, Peking University ShouGang Hospital, Beijing, P.R. China
\end{abstract}

Received June 24, 2010; Accepted August 17, 2010

DOI: 10.3892/ijo_00000802

\begin{abstract}
The tumor suppressor REIC/Dkk-3 is a secretory protein which was originally identified to be downregulated in human immortalized cells. In the present study, we investigated the expression pattern of REIC/Dkk-3 in various cell types to characterize its physiological functions. We first examined the expression level of REIC/Dkk-3 in a broad range of cancer cell types and confirmed that it was significantly downregulated in all of the cell types. We also examined the tissue distribution pattern in a variety of normal mouse organs. Ubiquitous REIC/Dkk-3 protein expression was observed in the organs. The expression was abundant in the liver, heart and brain tissue, but was absent in the spleen and peripheral blood mononuclear cells. The immunohistochemical analyses revealed that the subcellular localization of REIC/Dkk-3 had a punctate pattern around the nucleus, indicating its association with secretory vesicles. In cancer cells stably transfected with REIC/Dkk-3, the protein was predominantly localized to the endoplasmic reticulum (ER) under observation with confocal microscopy. Because REIC/ Dkk-3 was found to be abundantly expressed in the acinar epithelial cells of the mouse prostate, we analyzed the effects of recombinant REIC/Dkk-3 protein on the acinar morphogenesis of RWPE-1 cells, which are derived from human normal prostate epithelium. Statistically significant acinar growth was observed in the culture condition with $10 \mu \mathrm{g} / \mathrm{ml}$
\end{abstract}

Correspondence to: Dr Masami Watanabe, Department of Urology, Graduate School of Medicine, Dentistry and Pharmaceutical Sciences, Okayama University, 2-5-1 Shikata-cho, Okayama 700-8558, Japan E-mail: mwcorrespondence@gmail.com

Key words: REIC/Dkk-3, tissue distribution, subcellular localization, prostate, acinar development
REIC/Dkk-3 protein, implicating the soluble form in prostatic acinar development. Current results suggest that REIC/Dkk-3 may play a role in regulating the morphological process of normal tissue architecture through an autocrine and/or paracrine manner.

\section{Introduction}

In a previous study using the subtractive hybridization technique to screen for genes expressed by normal but not by an immortalized cell line, we identified multiple mRNA transcripts encoding putative candidate tumor suppressor genes. One of these mRNAs encoded a protein with glycosylation sites and signal peptide, and was named REIC (reduced expression in immortalized cells) (1). By a homology search, the REIC gene was found to be identical to the Dickkopf-3 (Dkk-3) gene, which is a member of the Dickkopf gene family. Several investigators have demonstrated the anti-tumor activities of this gene; ectopic REIC/Dkk-3 expression induced apoptosis in cancer cells and inhibited the cellular growth and invasion of these cells (2-12). The forced expression of REIC/Dkk-3 using a plasmid vector inhibited cell growth in HeLa and liver cancer cell lines (12). An adenoviral vector carrying REIC/Dkk-3 selectively induced apoptosis in prostate and testicular cancer cells, but not in non-cancerous cells, through the activation of c-Jun- $\mathrm{NH}_{2}$-kinase (JNK) and c-Jun $(8,11)$. A recent study in malignant mesothelioma cells indicated that adenovirus-mediated REIC/Dkk-3 overexpression triggered the significant induction of apoptosis via endoplasmic reticulum (ER) stress (5). Ad-REIC treatment also inhibited the expression of Id-1, which is involved in cell cycle progression and anti-apoptosis (5). Therefore, accumulating evidence suggests that the intracellular overexpression of REIC/Dkk-3 plays a distinct role against oncogenesis.

REIC/Dkk-3 is a secretory protein (1) and at present, little is known about its physiological role in normal cells. Recently, 
our study demonstrated that recombinant REIC/Dkk-3 protein induced the differentiation of human peripheral blood monocytes into a novel cell type with features of dendritic cells generated by IL-4 and GM-CSF treatment (2). Both cell populations induced by REIC/Dkk-3 protein or IL-4+GMCSF treatment exhibited similar proportions of CD11c+, CD40+, CD86+ and HLA-DR+ cells and endocytic capacity, suggesting similar functions of soluble REIC/Dkk-3 with these cytokines. Moreover, an analysis of the signal transducers and activators of transcription (STAT) pathways revealed that the REIC/Dkk-3 protein induced phosphorylation of STAT1 and STAT3 in the treated monocytes. Thus, REIC/ Dkk-3 protein possesses cytokine-like activity in peripheral blood monocytes and is involved in the cellular differentiation to a dendritic cell-like phenotype. These observations suggest a putative role of REIC/Dkk-3 protein in the morphogenic processes of specific normal cells.

The distribution, location, and subcellular localization of REIC/Dkk-3 protein in normal cells are of particular importance in its biological activities. We herein provide evidence that REIC/Dkk-3 is ubiquitously expressed in different mouse organs, and is subcellularly localized to the endoplasmic reticulum. To elucidate the physiological function, we further investigated the activity of recombinant REIC/Dkk-3 protein in vitro during the acinar morphogenesis of prostate-derived epithelial cells.

\section{Materials and methods}

Cell culture. Normal human prostate epithelial cells (PrEC) and prostate stromal cells (PrSC) were purchased from Lonza (Basel, Switzerland) and were cultivated using medium recommended by the supplier. The prostate cancer cell lines PC3, DU145, and LNCaP and the other human cell lines were provided by the American Type Culture Collection (Rockville, MD). Normal human fibroblasts (OUMS24) were established by the Department of Cell Biology in our university (11). Dulbecco's modified Eagle's medium (DMEM; Invitrogen, Carlsbad, CA), RPMI-1640 and HAM'S F-12 K medium (Nissui, Tokyo, Japan) were used for the culture of human cancer cell lines and OUMS24 cells. The RM9 mouse prostate cancer cell line was kindly provided by Dr T.C. Thompson (The University of Texas, M.D. Anderson Cancer Center, Houston, TX). This cell line was derived from a primary prostate tumor induced in the Zipras/myc-9-infected mouse prostate reconstitution (MPR) model system using C57BL/6 mice (13). The cancer cell lines were grown in medium supplemented with $10 \%$ fetal bovine serum (FBS; Biowest, Nuaille, France), penicillin $(50 \mathrm{IU} / \mathrm{ml})$ and streptomycin $(50 \mu \mathrm{g} / \mathrm{ml})$ under a humidified atmosphere of $5 \% \mathrm{CO}_{2}$ at $37^{\circ} \mathrm{C}$.

Stable transfection of RM9 cells with REIC/Dkk-3 gene. A panel of RM9 cell clones expressing REIC/Dkk-3 was established by stable transfection with a sense expression plasmid (14). A plasmid of pcDNA3 (Invitrogen) containing the human REIC/Dkk-3 gene and the selectable marker gene for neomycin resistance was used to generate REIC/Dkk-3expressing stable clones. As a control, RM9 cells were transfected with the pcDNA3 vector. Transfections were performed using the Lipofectamine reagent (Invitrogen) according to the manufacturer's protocol. The cells were transfected with each plasmid for $6 \mathrm{~h}$ in a serum-free medium, and the medium was exchanged for fresh complete medium. At $48 \mathrm{~h}$ after transfection, cells were placed into selection medium containing $500 \mu \mathrm{g} / \mathrm{ml} \mathrm{G} 418$ (Sigma, St. Louis, MO). After 21 days of selection, individual G418-resistant colonies were subcloned and the stable transfectants were stored in liquid nitrogen.

Reverse transcriptase-polymerase chain reaction (RT-PCR). Total RNA was isolated using the RNeasy Mini kit (Qiagen, Valencia, CA, USA), and cDNA synthesis was performed using M-MLV reverse transcriptase (Invitrogen), both according to the manufacturers' recommendations. The linear amplification ranges for the REIC/Dkk-3 and B-actin genes were examined during serial PCR cycles, and the optimal numbers of PCR cycles were determined. The sequences of forward and reverse primers were used as follows: REIC/ Dkk-3: 5'-ATGCAGCGGCTTGGGGCCACCCTGCTG TGC-3', 5'-GATGGTCCCATTGCTGCCCCTGGTGGC CAT-3', ß-actin: 5'-TGTATGCCTCTGGTCGTACCAC-3', 5'-ACAGAGTACTTGCGCTCAGGAG-3'. After RT-PCR, $5 \mu 1$-aliquots of the products were separated on a $2 \%$ agarose gel by electrophoresis and were stained with ethidium bromide.

Immunoblot analysis. The cells were lysed in ice-cold lysis buffer [20 mM Tris (pH 7.5), $150 \mathrm{mM} \mathrm{NaCl}, 1$ mM Na 2 EDTA, $1 \mathrm{mM}$ EGTA, $1 \%$ Triton, $2.5 \mathrm{mM}$ sodium pyrophosphate, $1 \mathrm{mM}$ ß-glycerophosphate, $1 \mathrm{mM} \mathrm{Na} \mathrm{VO}_{4}, 1 \mu \mathrm{g} / \mathrm{ml}$ leupeptin] and were incubated for $15 \mathrm{~min}$ at $4^{\circ} \mathrm{C}$. Mouse tissue was homogenized in the same ice-cold lysis buffer. Insoluble fragments were removed by centrifugation at $16,000 \mathrm{x} \mathrm{g}$ for $10 \mathrm{~min}$ at $4^{\circ} \mathrm{C}$, and the supernatant lysate was immediately flash-frozen and stored at $-80^{\circ} \mathrm{C}$. The protein concentrations were determined using a protein assay kit (Bio-Rad, Hercules, CA). Approximately $10 \mu \mathrm{g}$ of the extracted protein was mixed with 6X loading buffer [450 mM Tris ( $\mathrm{pH} 6.8), 45 \%$ sucrose, $10 \%$ ß-mercaptoethanol, 15\% SDS, and bromophenol blue] and was separated on SDS-PAGE gradient gels (BioRad). Proteins were electrotransferred onto PVDF membranes in $25 \mathrm{mM}$ Tris, $192 \mathrm{mM}$ glycine and $20 \%$ ethanol. Following the transfer, membranes were blocked with $5 \%$ non-fat dry milk in phosphate-buffered saline- $0.1 \%$ Tween-20 and were probed with the antibodies as follows. A rabbit polyclonal anti-REIC/Dkk-3 antibody was previously made in our laboratory (11). Polyclonal antibodies against human Dkk-1 (AF1096, R and D systems, Minneapolis, MN), Dkk-2 (AP1522a, ABGENT, San Diego, CA), Dkk-4 (AP1524b, ABGENT), and a mouse monoclonal antibody against tubulin (T6074, Sigma) were purchased. Horseradish peroxidaseconjugated secondary antibodies and the ECL substrate kit (GE Healthcare, Buckinghamshire, UK) were used for the detection of antibody-bound proteins.

Immunostaining. For the immunohistological analysis of the REIC/Dkk-3 expression in normal mouse organs, mice were sacrificed and various tissues were removed and fixed with $4 \%$ paraformaldehyde in $100 \mathrm{mM}$ phosphate buffer $(\mathrm{pH} 7.4)$ for $16 \mathrm{~h}$ at $4^{\circ} \mathrm{C}$. The tissues were then embedded in the OCT compound and snap-frozen in liquid nitrogen. The frozen sections $(10 \mu \mathrm{m})$ were permeabilized with PBS containing 


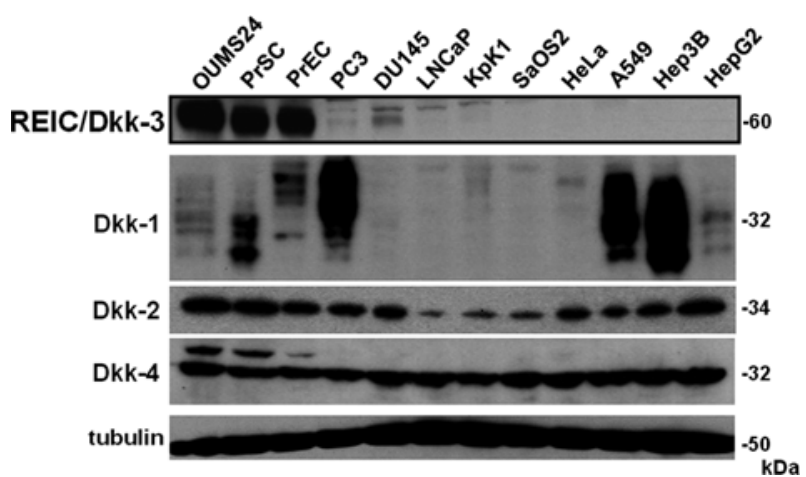

Figure 1. The expression levels of the Dkks (REIC/Dkk-3, Dkk-1, Dkk-2 Dkk-4) were determined by an immunoblot analysis in normal human cells (OUMS24: fibroblast-derived cell line, PrSC: prostate stromal cells, PrEC: prostate epithelial cells) and in human cancer-derived cell lines (PC3, DU145, LNCaP: prostate cancer, KpK1: renal cancer, SaOS2: osteosarcoma, HeLa: cervical cancer, A549: lung cancer, Hep3B, HepG2: hepatoblastoma cell lines). Tubulin was used as a loading control.

$0.1 \%$ Triton $\mathrm{X}-100$ and were immunohistochemically stained as previously described (15). Endogenous peroxidase activity was blocked by incubation with $3 \%$ hydrogen peroxide in methanol for $10 \mathrm{~min}$. The sections were incubated with the rabbit polyclonal anti-REIC/Dkk-3 antibody (1:50 dilution in PBS) at room temperature (RT) for $16 \mathrm{~h}$ and were then incubated with the Histofine Simple Stain MAX PO (Nichirei, Tokyo, Japan) for $30 \mathrm{~min}$. Bound antibodies were detected using diaminobenzidine-tetrahydrochloride as the substrate and thereafter the sections were counterstained with hematoxylin.

Immunocytochemical staining of REIC/Dkk-3 in RM9 control and REIC/Dkk-3 clone cells was carried out by costaining the endoplasmic reticulum organelle. Cells were plated and cultured to $30-40 \%$ confluence on a coated $12-\mathrm{mm}$ coverslip (Asahi Glass Co., Chiba, Japan) in 24-well plates. The cells were fixed with $4 \%$ paraformaldehyde in $100 \mathrm{mM}$ phosphate buffer ( $\mathrm{pH} \mathrm{7.4)}$ ) for $10 \mathrm{~min}$ at RT. The cells were incubated with $0.1 \%$ Triton-X in PBS for $10 \mathrm{~min}$ and were blocked with 3\% BSA in PBS for $1 \mathrm{~h}$. The cells were incubated with a goat polyclonal anti-REIC/Dkk-3 antibody (AF1118, R\&D Systems) for $2 \mathrm{~h}$ and then with Alexa488 green fluorescence-conjugated anti-goat secondary antibody (Molecular Probes, Eugene, OR) for $1 \mathrm{~h}$ at RT. To determine the distribution in the endoplasmic reticula, the cells were incubated with Alexa546 red fluorescence-conjugated concanavalin A (Molecular Probes) for 15 min at RT. To stain the cell nucleus, 4',6-diamidino-2-phenylindol-dihydrochloride (DAPI) was used (Kirkegaard \& Perry Laboratories, Gaithersburg, MD). The immunofluorescence staining was visualized by a confocal microscope system (CSU10; Yokogawa Electric, Kanazawa, Japan) and the images were analyzed with the MetaMorph software program (Molecular Devices, Sunnyvale, CA).

Preparation of recombinant human REIC/Dkk-3 protein. Human REIC/Dkk-3 stable expression clones in $\mathrm{CHO}$ cells were established by electroporated transfection of the plasmids encoding the full-length human REIC/Dkk-3 gene with a histidine tag and a puromycin-resistant gene (2). A total of $10^{7}$ cells was cultured in 1 liter of $\mathrm{CHO}$ protein-free medium
(C5467, Sigma, St. Louis, MO) with $2 \mathrm{mM}$ L-glutamine and $8 \mu \mathrm{M}$ puromycin at $37^{\circ} \mathrm{C}$ in $5 \% \mathrm{CO}_{2}$ for 1 week with gentle shaking. Using the TALON Resin (no. 635501, Clontech Laboratories, Mountain View, CA) in the culture supernatant, histidine-tagged human REIC/Dkk-3 protein was collected and subjected to Fast Protein Liquid Chromatography (MonoQ5/50GL column, GE Healthcare, Buckinghamshire, UK). The purified human REIC/Dkk-3 protein was dialyzed with phosphate-buffered saline (PBS) and the stock solutions of the protein were maintained at $-80^{\circ} \mathrm{C}$ until use.

To determine whether the recombinant REIC/Dkk-3 protein was glycosylated, a protein deglycosylation assay was performed. Recombinant REIC/Dkk-3 protein $(5 \mu \mathrm{g})$ was treated with $2 \mathrm{U} \mathrm{N}$-glycosidase or $2 \mathrm{mU}$ O-glycosidase, or both, according to the manufacturer's instructions (Roche Applied Science, Mannheim, Germany). A total of $1 \mu \mathrm{g}$ REIC/Dkk-3 protein was separated by electrophoresis in SDS-PAGE gel followed by Coomassie Brilliant Blue (C.B.B.) staining.

Acinar morphogenesis assay. The three-dimensional (3D) culture of RWPE-1 cells on Matrigel was carried out as described by Kawano et al (16), with minor modifications. RWPE-1 cells, which were derived from human normal prostate epithelium, were grown in keratinocyte serum-free medium (SFM) supplemented with bovine pituitary extract and EGF (Invitrogen). Eight-well glass chamber slides were coated with $40 \mu \mathrm{l}$ of Phenol Red-free BD Matrigel (BD Biosciences) per well, herein referred to as Matrigel. RWPE-1 cells were trypsinized and resuspended in keratinocyte SFM with complete supplements. Five thousand cells were plated per well in assay medium (keratinocyte SFM with complete supplements and 2\% Matrigel) with recombinant REIC/Dkk-3 protein at the indicated concentrations. After 4 days of incubation, images were randomly obtained using a microscope and the average acinar diameter was analyzed in each treatment group.

Statistical analysis. The data are shown as the mean \pm SE. Student's unpaired t-test was performed for the statistical analyses between the two groups, and differences with $\mathrm{p}<0.05$ were considered to be statistically significant.

\section{Results}

The expression of REIC/Dkk-3 protein is significantly downregulated in a broad range of cancer cell types. We first examined REIC/Dkk-3 expression in various cell lines, including human prostate cancer cells (Fig. 1). In normal human fibroblasts (OUMS24), prostate epithelial (PrEC) and stromal (PrSC) cells, REIC/Dkk-3 protein was detected as single major band around $60 \mathrm{kDa}$ in size by an immunoblot analysis. REIC/Dkk-3 protein was barely detectable in three prostate cancer cell lines and in other cancer cell lines of other origins. We also examined the expression of the other Dkk family proteins, including Dkk-1, Dkk-2 and Dkk-4, in these cell lines. Strong Dkk-1 expression was observed in the PC3, A549 and Hep3B cancer cell lines. The broad-ranged expression of Dkk-2 and Dkk-4 was observed in the examined cancer cell lines. 


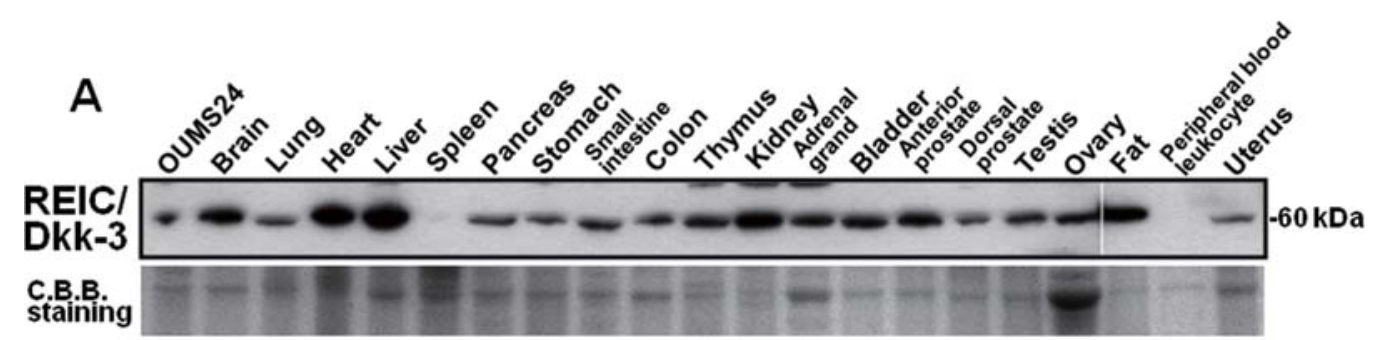

B
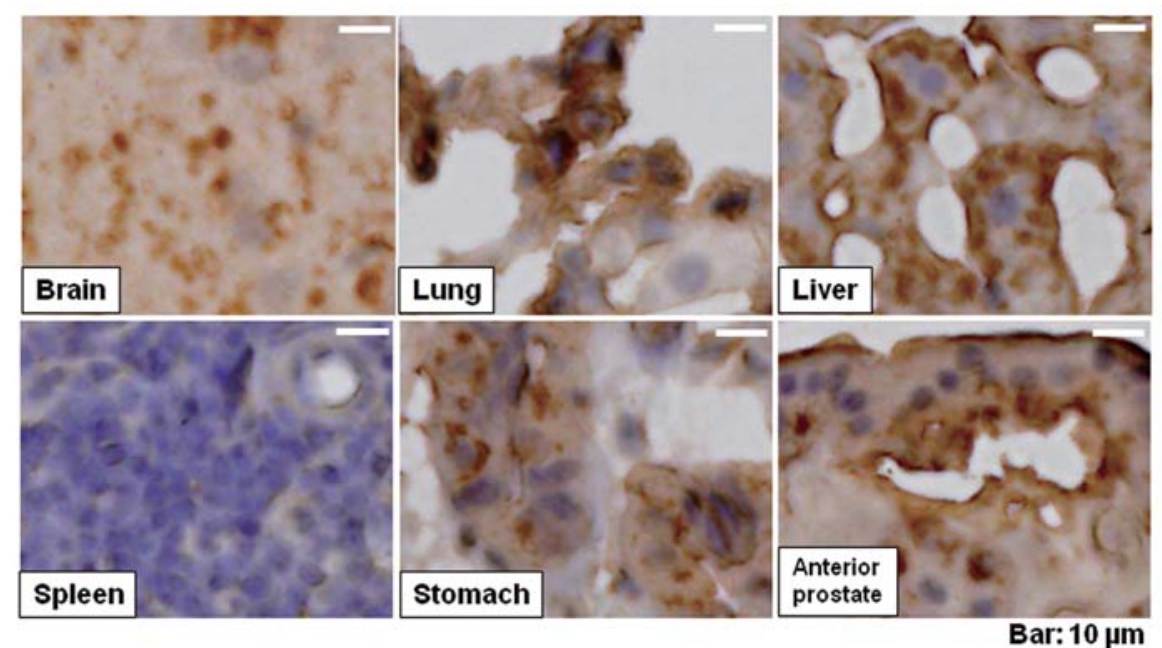

Figure 2. (A) The tissue distribution of REIC/Dkk-3 was analyzed by an immunoblot analysis in the organs of mice. Coomassie Brilliant Blue (C.B.B.) staining of the membrane is shown as a loading control. (B) A normal tissue histological analysis of REIC/Dkk-3 was performed for tissue samples of brain, lung, liver, spleen, stomach, and the anterior prostate. The immunohistochemical staining was developed using diaminobenzidine-tetrahydrochloride as the substrate, and the tissue slices were counterstained with hematoxylin.

REIC/Dkk-3 protein is ubiquitouly expressed in mouse organs. The distribution pattern of REIC/Dkk-3 was examined by immunoblotting in a number of mouse organs (Fig. 2A). Relatively strong expression of REIC/Dkk-3 protein was observed in the liver, heart and brain but was minimally detected in the spleen and peripheral blood leukocytes. The REIC/Dkk-3 expression was ubiquitously observed in the other organs examined, including the prostate.

The immunolocalization of REIC/Dkk-3 in various mouse organs was also examined using a REIC/Dkk-3-specific antibody, and representative results are shown in Fig. 2B. As expected from the immunoblot results, positive staining of REIC/Dkk-3 was detected in the ubiquitous organs. The staining pattern was punctate in the cellullar cytoplasm, and this finding was clearly observed in brain and liver. The simple columnar epithelia of the gastrointestinal tract were also stained for REIC/Dkk-3. In the stomach, REIC/Dkk-3 staining was observed as dots in the glandular epithelial cells of the mucosa. The luminal epithelial cells of the prostate were also stained for REIC/Dkk-3, and the signal was typically observed in the cytoplasm which faced the lumen of the gland. In the testis, positive staining of REIC/Dkk-3 was present in the Leydig cells at the interstitial space, whereas no immunostaining was observed at the seminiferous epithelium which consists of germ cells and Sertoli cells (data not shown). REIC/Dkk-3 was also present in the intralobular duct epithelia of the pancreas and kidney tubule epithelia (data not shown). Little or no staining of any tissue sections was observed using the affinity-purified control rabbit polyclonal $\operatorname{IgG}$ preparation. We did not observe any positive staining for REIC/Dkk-3 in the spleen, which was consistent with the immunoblotting results.

REIC/Dkk-3 protein is predominantly localized to the endoplasmic reticulum in stable transfected cells. To further investigate the subcellular distribution of the REIC/Dkk-3 protein, we established the REIC/Dkk-3 stably-transfected mouse prostate cancer cells. REIC/Dkk-3 expression in the REIC/Dkk-3-transfected RM9 clones, the parental and control mock clone was confirmed by a RT-PCR analysis (Fig. 3A). An immunofluorescence analysis was thereafter conducted in the REIC/Dkk-3 and control transfectants. In the REIC/Dkk-3 transfectant, REIC/Dkk-3 staining formed a punctate pattern in the cellular cytoplasm. No staining signal of REIC/Dkk-3 was detected in the control transfectants. The punctate distribution of REIC/Dkk-3 in the cytoplasm was consistent with the cellular localization pattern in multiple mouse organs. These findings suggest that REIC/Dkk-3 protein localizes in organelles such as the endosomes and endoplasmic reticulum. We performed co-staining experiments utilizing fluorescenceconjugated concanavalin A, a marker specific for the endoplasmic reticulum, to confirm the localization of REIC/Dkk-3 at the cellular compartments. REIC/Dkk-3 protein significantly co-localized with the organelle marker (Fig. 3B), thus indicating its subcellular localization at the endoplasmic reticulum.

Recombinant REIC/Dkk-3 protein promoted acinar development in the RWPE-1 prostatic epithelial cells. An amino-acid sequence analysis indicated that REIC/Dkk-3 possesses a signal peptide (amino acid 1-22) in its $\mathrm{N}$-terminal domain 
A

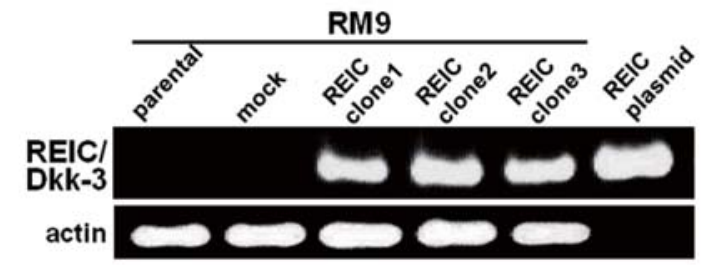

B

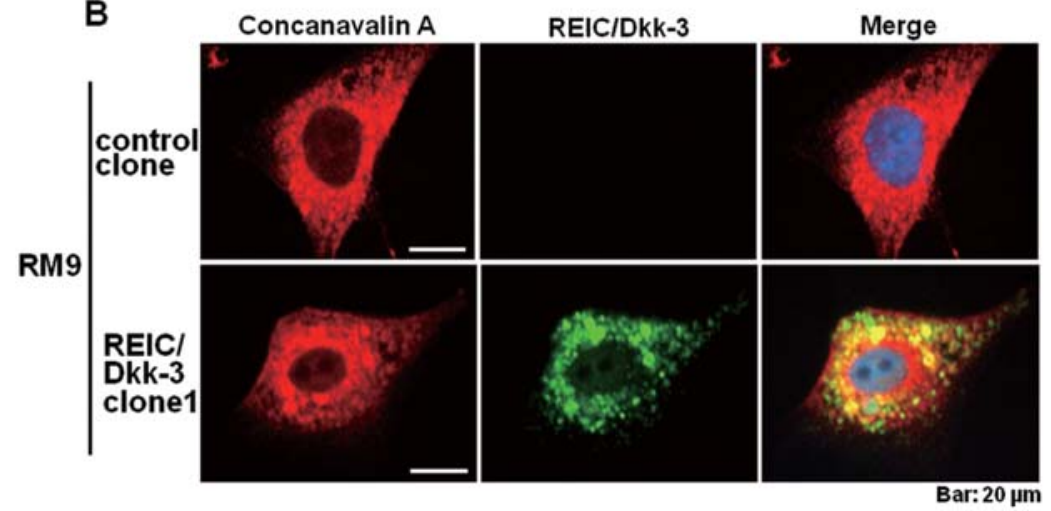

Figure 3. (A) The relative expression levels of REIC/Dkk-3 were analyzed by a semiquantitative RT-PCR analysis in RM9 clones stably transfected with REIC/Dkk-3 and control cells. B-actin was examined as an internal control. (B) The co-localization of REIC/Dkk-3 and concanavalin A (endoplasmic reticulum marker) was examined by double immunofluorescence staining and was imaged by confocal microscopy. Images in red and green show the subcellular localization of concanavalin A and REIC/Dkk-3, respectively. The areas of overlap between the endoplasmic reticulum and REIC/Dkk-3 are shown in yellow.
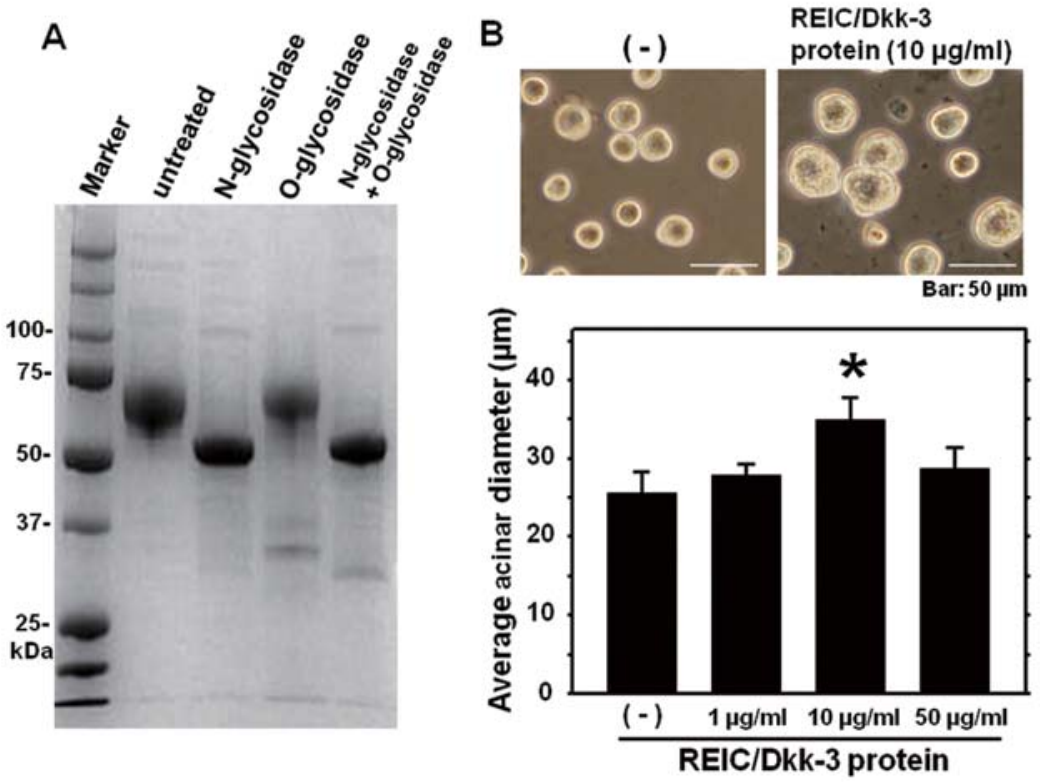

Figure 4. (A) Recombinant REIC/Dkk-3 was analyzed for glycosylation status. The protein sample was deglycosylated with N-glycosidase or O-glycosidase and was separated by electrophoresis by SDS-PAGE. The gel was stained with Coomassie Brilliant Blue. (B) Three-dimensional (3D) culture of RWPE-1 cells on Matrigel was carried out in an acinar morphogenesis assay. The recombinant REIC/Dkk-3 protein was added to the medium at the indicated concentrations. After 4 days of incubation, the morphology of acinar-like structures formed by RWPE-1 cells was observed under a microscope and a representative image is shown. The lower panel indicates the average acinar diameter measured in each treatment group. Four independent experiments were performed for each group. A significant difference was observed in comparison to the non-treatment group.

and three potential $\mathrm{N}$-linked glycosylation sites (at amino acids 96-99, 106-109 and 204-207), suggestive of a secreted $\mathrm{N}$-glycosylated protein (1). These data suggest the intracellular localization of REIC/Dkk-3 protein in the endoplasmic reticulum, and that the soluble secreted form of the protein may exert its physiological functions. To investigate the role of the REIC/Dkk-3 protein, full-length human REIC/Dkk-3 protein was generated in $\mathrm{CHO}$ cells. The purified recombinant REIC/Dkk-3 protein was recognized as single major band approximately $65 \mathrm{kDa}$ in size by gel electrophoresis followed by C.B.B. staining, and the purity was confirmed to be greater than $95 \%$ (Fig. 4A). To examine whether the recombinant REIC/Dkk-3 was glycosylated, the protein was treated with $\mathrm{N}$-glycosidase, O-glycosidase and both. The 
molecular weight was reduced when treated with N-glycosidase, but it was not reduced further when additionally treated with O-glycosidase, which was therefore indicative of the presence of $\mathrm{N}$-glycosylation sites in the protein.

To examine the possibility that REIC/Dkk-3 is involved in prostate gland differentiation, we utilized a three-dimensional (3D) in vitro model of human prostate acinar formation using RWPE-1 cells. RWPE-1 cells undergo acinar-like morphogenesis with spherically-arranged polarization and both structural and functional differentiation when grown in a 3D Matrigel environment (16). RWPE-1 cells were grown in keratinocyte serum-free medium with supplements for 4 days, and the cells thereafter organized themselves into distinct acini (Fig. 4B). When RWPE-1 cells were treated with recombinant REIC/Dkk-3 protein $(10 \mu \mathrm{g} / \mathrm{ml})$, the cells developed larger acini compared to the untreated cells (Fig. 4B). The result indicates that soluble form of REIC/Dkk-3 protein is involved in the acinar development and supports the possibility that REIC/Dkk-3 protein plays a role in the normal differentiation of the prostate gland.

\section{Discussion}

We herein demonstrated that the expression of REIC/Dkk-3 is significantly downregulated in various human cancer cell lines. The cancer-specific downregulation was observed only for REIC/Dkk-3, but not of the other Dkks. The immunoblot analyses clearly show that REIC/Dkk-3 is ubiquitously expressed in a variety of mouse organs. REIC/Dkk-3 expression was seen at higher levels in the liver, heart, kidney and brain but was not observed in the spleen and peripheral blood leukocytes. This expression pattern was consistent with a previous examination in various human tissues (1). These findings suggest that the REIC/Dkk-3 protein has physiological roles in normal cells, and its expression may be suppressed during cancer development.

To clarify the localization of the REIC/Dkk-3 protein in various normal tissues, an immunostaining analysis was performed. The staining pattern of REIC/Dkk-3 was punctate at the cellular cytoplasm in the brain and liver. In the prostatic epithelium, immunoreactivity was observed near the cell surface of the inner lumen of the gland. These histological results indicated that the REIC/Dkk-3 protein is a secreted protein and its soluble form localizes in membranous cellular organelles such as the endoplasmic reticulum and the Golgi apparatus. Thereafter, the subcellular localization of REIC/ Dkk-3 was analyzed in the stably-transfected mouse prostate cancer RM9 cells. As expected, the REIC/Dkk-3 protein was predominantly localized to the endoplasmic reticulum.

There have been few reports investigating the physiological functions of secreted or exogenous REIC/Dkk-3. It was previously demonstrated that a loss of REIC/Dkk-3 expression was significantly correlated with atypical prostatic differentiation and prostate cancer progression $(11,16)$. Considering the fact that REIC/Dkk-3 is a secreted protein and is localized near the cell surface of prostate epithelial cells which form the acinar, we speculated that paracrine REIC/Dkk-3 signaling has a physiological role in glandular polarization and/or differentiation. Therefore, to elucidate the biological function of REIC/Dkk-3, we performed an acinar morphogenesis assay using the RWPE-1 cell-3D model. The addition of REIC/Dkk-3 to the culture medium of RWPE-1 cells resulted in an increase in acinar size, indicating that REIC/Dkk-3 protein is important for acinar differentiation and development. This finding suggested that extracellular REIC/Dkk-3 protein affects the cellular activities necessary for the development of the normal acinar architecture. Interestingly, when a bladder cancer cell line was treated with REIC/Dkk-3, REIC/Dkk-3 inhibited the invasiveness and motility in vitro (7). In addition, in vitro studies with a prostate cancer cell line indicated that overexpression of REIC/Dkk-3 inhibited cell invasion/migration with reduced matrix metalloproteinase-2 activity (9). These observations suggest that REIC/Dkk-3 modifies the cell motility and migration of the luminal cells during acinar development and affect the acinar morphology.

The molecular function and mechanism by which REIC/ Dkk-3 protein exogenously acts are not presently understood. REIC/Dkk-3 is a member of the Dickkopf protein family, and is known to interfere with $\mathrm{Wnt} / \mathrm{ß}$-catenin signaling via Wnt receptors $(1,17,18)$. However, several studies have demonstrated that REIC/Dkk-3 does not affect the Wnt/Bcatenin signaling under these conditions $(16,17)$. We have recently noted the immunological role of the REIC/Dkk-3 protein in human monocyte differentiation (2). After incubation with REIC/Dkk-3 protein, the levels of phosphorylated STAT1 and STAT3 were significantly upregulated in differentiating monocytes. Because the activated STAT pathway is one of the major signaling pathways during cellular differentiation $(19,20)$, investigation based on these signaling events may elucidate the underlying molecular mechanism of REIC/Dkk-3 during prostate acinar development.

In conclusion, we have shown that REIC/Dkk-3 is expressed in a number of normal organs and is not expressed in the majority of cancer cell lines examined in the present series. We also observed that REIC/Dkk-3 is an N-glycosylated protein, and the majority of this protein localizes to the endoplasmic reticulum. In vitro assays suggested that the soluble form of REIC/Dkk-3 protein was associated with acinar development, thus suggesting that REIC/Dkk-3 is involved in prostate gland differentiation. These findings also suggested that reduced REIC/Dkk-3 may contribute to a disorganized development in various tissues. An important future step will be to clarify the biological functions and mechanisms of action of REIC/Dkk-3 in terms of the normal tissue differentiation and cancer development.

\section{Acknowledgements}

This study was supported by a grant from the Ministry of Education, Culture, Sports, Science and Technology's FY2006 'Creation of Innovation Centers for Advanced Interdisciplinary Research Areas' Scheme in Japan. We thank Katsuo Ohno (Okayama University) for his technical assistance.

\section{References}

1. Tsuji T, Miyazaki M, Sakaguchi M, Inoue Y and Namba M: A REIC gene shows down-regulation in human immortalized cells and human tumor-derived cell lines. Biochem Biophys Res Commun 268: 20-24, 2000. 
2. Watanabe M, Kashiwakura Y, Huang P, Ochiai K, Futami J, Li SA, Takaoka M, Nasu Y, Sakaguchi M, Huh NH and Kumon H: Immunological aspects of REIC/Dkk-3 in monocyte differentiation and tumor regression. Int J Oncol 34: 657-663, 2009

3. Sakaguchi M, Kataoka K, Abarzua F, Tanimoto R, Watanabe M, Murata H, Than SS, Kurose K, Kashiwakura Y, Ochiai K, Nasu Y, Kumon H and Huh NH: Overexpression of REIC/Dkk-3 in normal fibroblasts suppresses tumor growth via induction of IL-7. J Biol Chem 284: 14236-14244, 2009.

4. Kawasaki K, Watanabe M, Sakaguchi M, Ogasawara Y, Ochiai K, Nasu Y, Doihara H, Kashiwakura Y, Huh NH, Kumon H and Date H: REIC/Dkk-3 overexpression downregulates P-glycoprotein in multidrug-resistant MCF7/ADR cells and induces apoptosis in breast cancer. Cancer Gene Ther 16: 65-72, 2009.

5. Kashiwakura Y, Ochiai K, Watanabe M, Abarzua F, Sakaguchi M, Takaoka M, Tanimoto R, Nasu Y, Huh NH and Kumon H: Down-regulation of inhibition of differentiation-1 via activation of activating transcription factor 3 and Smad regulates REIC/Dickkopf-3-induced apoptosis. Cancer Res 68 : 8333-8341, 2008.

6. Mizobuchi Y, Matsuzaki K, Kuwayama K, Kitazato K, Mure H, Kageji T and Nagahiro S: REIC/Dkk-3 induces cell death in human malignant glioma. Neuro Oncol 10: 244-253, 2008.

7. Saeb-Parsy K, Veerakumarasivam A, Wallard MJ, Thorne N, Kawano Y, Murphy G, Neal DE, Mills IG and Kelly JD: MT1MMP regulates urothelial cell invasion via transcriptional regulation of Dickkopf-3. Br J Cancer 99: 663-669, 2008.

8. Tanimoto R, Abarzua F, Sakaguchi M, Takaishi M, Nasu Y, Kumon $\mathrm{H}$ and Huh NH: REIC/Dkk-3 as a potential gene therapeutic agent against human testicular cancer. Int J Mol Med 19: 363-368, 2007

9. Edamura K, Nasu Y, Takaishi M, Kobayashi T, Abarzua F, Sakaguchi M, Kashiwakura Y, Ebara S, Saika T, Watanabe M, Huh NH and Kumon H: Adenovirus-mediated REIC/Dkk-3 gene transfer inhibits tumor growth and metastasis in an orthotopic prostate cancer model. Cancer Gene Ther 14: 765-772, 2007.

10. Abarzua F, Sakaguchi M, Tanimoto R, Sonegawa H, Li DW, Edamura K, Kobayashi T, Watanabe M, Kashiwakura Y, Kaku H, Saika T, Nakamura K, Nasu Y, Kumon H and Huh NH: Heat shock proteins play a crucial role in tumor-specific apoptosis by REIC/Dkk-3. Int J Mol Med 20: 37-43, 2007.
11. Abarzua F, Sakaguchi M, Takaishi M, Nasu Y, Kurose K, Ebara S, Miyazaki M, Namba M, Kumon $\mathrm{H}$ and Huh NH: Adenovirus-mediated overexpression of REIC/Dkk-3 selectively induces apoptosis in human prostate cancer cells through activation of c-Jun-NH2-kinase. Cancer Res 65: 9617-9622, 2005.

12. Hsieh SY, Hsieh PS, Chiu CT and Chen WY: Dickkopf-3/REIC functions as a suppressor gene of tumor growth. Oncogene 23: 9183-9189, 2004

13. Thompson TC, Southgate J, Kitchener G and Land H: Multistage carcinogenesis induced by ras and myc oncogenes in a reconstituted organ. Cell 56: 917-930, 1989.

14. Chen J, Watanabe M, Huang P, Sakaguchi M, Ochiai K, Nasu Y, Ouchida M, Huh NH, Shimizu K, Kashiwakura Y, Kaku H and Kumon H: REIC/Dkk-3 stable transfection reduces the malignant phenotype of mouse prostate cancer RM9 cells. Int J Mol Med 24: 789-794, 2009

15. Watanabe M, Kashiwakura Y, Kusumi N, Tamayose K, Nasu Y, Nagai A, Shimada T, Daida H and Kumon H: Adeno-associated virus-mediated human IL-10 gene transfer suppresses the development of experimental autoimmune orchitis. Gene Ther 12: 1126-1132, 2005.

16. Kawano Y, Kitaoka M, Hamada Y, Walker MM, Waxman J and Kypta RM: Regulation of prostate cell growth and morphogenesis by Dickkopf-3. Oncogene 25: 6528-6537, 2006.

17. Tsuji T, Nozaki I, Miyazaki M, Sakaguchi M, Pu H, Hamazaki Y, Iijima O and Namba M: Antiproliferative activity of REIC/Dkk-3 and its significant down-regulation in non-small-cell lung carcinomas. Biochem Biophys Res Commun 289: 257-263, 2001.

18. Glinka A, Wu W, Delius H, Monaghan AP, Blumenstock C and Niehrs C: Dickkopf-1 is a member of a new family of secreted proteins and functions in head induction. Nature 391: 357-362, 1998.

19. Ihle JN: The Janus protein tyrosine kinase family and its role in cytokine signaling. Adv Immunol 60: 1-35, 1995.

20. Nosaka $T$ and Kitamura T: Janus kinases (JAKs) and signal transducers and activators of transcription (STATs) in hematopoietic cells. Int J Hematol 71: 309-319, 2000. 